FACTA UNIVERSITATIS (NIŠ)

Ser. Math. Inform. Vol. 35, No 4 (2020), 1017-1030

https://doi.org/10.22190/FUMI2004017S

\title{
ON $f$-KENMOTSU MANIFOLDS AND THEIR SUBMANIFOLDS WITH QUARTER SYMMETRIC METRIC CONNECTIONS *
}

\author{
Avijit Sarkar and Nirmal Biswas
}

(C) 2020 by University of Niš, Serbia / Creative Commons Licence: CC BY-NC-ND

Abstract. The object of the present paper is to study invariant submanifolds of $f$ Kenmotsu manifolds with respect to quarter symmetric metric connections. Some necessary and sufficient conditions for such submanifolds to be totally geodesic have been deduced. Also we have constructed an example of a submanifold of a five-dimensional $f$-Kenmotsu manifold to justify our results.

Keywords: $f$-Kenmotsu manifold; quarter symmetric metric connection.

\section{Introduction}

In 1924, Friedman and Schouten [10] introduced the notion of semi-symmetric metric connections on a manifold and the notion of quarter symmetric metric connections was defined and studied by Golab [11]. The quarter-symmetric metric connections are generalizations of the semi-symmetric metric connections. A linear connection $\bar{\nabla}$ in a Riemannian manifold is said to be a quarter symmetric metric connection [11] if the torsion tensor $T$ defined by

$$
T(X, Y)=\bar{\nabla}_{X} Y-\bar{\nabla}_{Y} X-[X, Y]
$$

satisfies

$$
T(X, Y)=\eta(Y) \phi X-\eta(X) \phi Y
$$

for any vector field $X, Y$ on the manifold. Here $\eta$ is a 1 -form and $\phi$ is a $(1,1)$ tensor field. If $\phi X=X$, then the quarter symmetric connection is reduced to a semi-symmetric connection. If the quarter symmetric connection $\bar{\nabla}$ satisfies the condition

$$
\left(\bar{\nabla}_{X} g\right)(Y, Z)=0
$$

for any vector field $X, Y, Z$ on the manifold, then the connection $\bar{\nabla}$ is said to be quarter symmetric metric connection; otherwise, it is non-metric connection.

Received August 15, 2020; accepted September 24, 2020

2020 Mathematics Subject Classification. Primary 53 C15; Secondary 53 D 25.

* The second author is supported by UGC, Id-421642. 
Quarter symmetric connections have been characterized by several authors ([3], [16], [17], [18], [26], [28]). Recently, P-Sasakian manifolds admitting a quarter symmetric metric connections have been studied by De et all [7].

The notion of invariant submanifolds is an important topic of study in differential geometry. If in a submanifold of an almost contact manifold the structure tensor maps tangent vector fields to tangent vector fields, then the submanifold is called invariant [5]. Invariant submanifolds of Sasakian manifolds were studied by M. Kon [14]. Invariant submanifolds of contact and para contact manifolds have been studied by several authors ( [8], [20], [21], [24], [25], [30]).

In 1982, Olszak and Rosca [22] introduced $f$-Kenmotsu manifolds and gave their geometric interpretations, they also proved that a Ricci symmetric $f$-Kenmotsu manifold is an Einstein manifold. Several authors ([4], [6], [29]) studied $f$-Kenmotsu manifolds. In the present paper we would like to study invariant submanifolds of $f$-Kenmotsu manifolds with respect to quarter symmetric metric connections. In fact, we have obtained the conditions for such submanifolds to be totally geodesic. The present paper is organized as follows:

Section 1, is introductory. After preliminaries in Section 2, we obtain the relations between the curvature tensor, Ricci tensor and scalar curvature of the manifold with respect to Levi-Civita connection and quarter symmetric metric connection in Section 3. Next we study invariant submanifolds of an $f$-Kenmotsu manifold and construct an example of a submanifold of a five-dimensional $f$-Kenmotsu manifold to justify our results. Finally, we obtain the conditions for such submanifolds to be totally geodesic.

\section{Preliminaries}

Let $\widetilde{M}$ be a $(2 n+1)$-dimensional differentiable manifold with an almost contact metric structure $(\phi, \xi, \eta, g)$, where $\phi$ is a $(1,1)$-tensor field, $\xi$ is a vector field, $\eta$ is a 1 -form and $g$ is the Riemannian metric on the manifold, satisfying the relations

$$
\begin{gathered}
\phi^{2} X=-X+\eta(X) \xi, \quad \eta(\xi)=1, \\
\eta(X)=g(X, \xi), \\
g(\phi X, \phi Y)=g(X, Y)-\eta(X) \eta(Y), \\
\phi \xi=0, \quad \eta \circ \phi=0, \quad g(X, \phi Y)=-g(\phi X, Y)
\end{gathered}
$$

for any vector fields $X, Y$ on the manifold $\widetilde{M}$.

The manifold $\widetilde{M}$ is called an $f$-Kenmotsu manifold if the covariant differentiation of $\phi$ satisfies the relation [29]

$$
\left(\widetilde{\nabla}_{X} \phi\right) Y=f(g(\phi X, Y) \xi-\eta(Y) \phi X)
$$


where $\widetilde{\nabla}$ is the Levi-Civita connection of the $f$-Kenmotsu manifolds and $f$ is a $C^{\infty}$-function on the manifold. If $f=\beta=$ constant $\neq 0$, then the manifold is $\beta$ Kenmotsu manifold [13] and if $f=0$, then the manifold reduces to cosymplectic manifold [13]. Moreover $f$-Kenmotsu manifold is called regular if $f^{2}+f^{\prime} \neq 0$, where $f^{\prime}=\xi f$.

Form (2.2), we get

$$
\widetilde{\nabla}_{X} \xi=f(X-\eta(X) \xi)
$$

Let $M^{2 m+1}(m<n)$ be a submanifold of a contact metric manifold $\widetilde{M}^{2 n+1}$. Let $\nabla$ and $\widetilde{\nabla}$ be the Levi-Civita connections of $M$ and $\widetilde{M}$, respectively. Then for any vector fields $X, Y \in \chi(M)$, the second fundamental form $h$ is defined by

$$
\widetilde{\nabla}_{X} Y=\nabla_{X} Y+h(X, Y)
$$

and for any vector field $V$ of normal bundle $T^{\perp} M$

$$
\widetilde{\nabla}_{X} V=-A_{V} X+\nabla_{X}^{\perp} V
$$

The second fundamental form $h$ and the shape operator $A_{V}$ are related by [27]

$$
g(h(X, Y), V)=g\left(A_{V} X, Y\right) .
$$

A submanifold $M$ of a $f$-Kenmotsu manifold is said to be totally umbilical if

$$
h(X, Y)=g(X, Y) H
$$

for any vector field $X, Y$ on $M ; H$ is the mean curvature of $M$ given by

$$
H=\frac{1}{2 m+1} \sum_{i=1}^{2 m+1} h\left(e_{i}, e_{i}\right) .
$$

Moreover, if $h(X, Y)=0$ for all $X, Y \in \chi(M)$, then the submanifold is called totally geodesic. If $H=0$, then the submanifold $M$ is minimal in $\widetilde{M}$.

Covariant derivative of order $p, p \geq 1$ of a $(0, k)$ tensor field is denoted by $\nabla^{p} T$. According to [23] the tensor $T$ is said to be recurrent and 2-recurrent if

and

$$
\begin{array}{r}
\left(\nabla^{2} T\right)\left(X_{1}, X_{2}, \ldots, X_{k} ; X, Y\right) T\left(Y_{1}, Y_{2}, \ldots Y_{k}\right)= \\
\left(\nabla^{2} T\right)\left(Y_{1}, Y_{2}, \ldots Y_{k} ; X, Y\right) T\left(X_{1}, X_{2}, \ldots, X_{k}\right)
\end{array}
$$

where $X, Y, X_{1}, X_{2}, \ldots, X_{k}, Y_{1}, Y_{2}, \ldots Y_{k} \in \chi(\widetilde{M})$. If $T$ is non-zero then there exists a unique 1 -form $\pi$ and a $(0,2)$ tensor $\psi$, such that

$$
\nabla T=T \otimes \pi, \quad \pi=d(\log \|T\|),
$$


and

$$
\nabla^{2} T=T \otimes \psi
$$

where $\|T\|=g(T, T)$.

In a $(2 n+1)$ dimensional $f$-Kenmotsu manifold, we have [22]

$$
\begin{gathered}
R(X, Y) \xi=f^{2}(\eta(X) Y-\eta(Y) X) \\
+(Y f) \phi^{2} X-(X f) \phi^{2} Y \\
S(X, \xi)=-\left(2 n f^{2}-\xi f\right) \eta(X)-(2 n-1) X f, \\
S(\xi, \xi)=-2 n\left(f^{2}-\xi f\right), \\
Q \xi=-\left(2 n f^{2}-\xi f\right) \xi-(2 n-1) \operatorname{grad} f,
\end{gathered}
$$

where $R, S$ and $Q$ denote the Riemann curvature tensor, Ricci tensor and Ricci operator respectively.

In a 3-dimensional $f$ - Kenmotsu manifold we also have [22]

$$
\begin{aligned}
R(X, Y) Z & =\left(\frac{r}{2}+2 f^{2}+2 f^{\prime}\right)(X \wedge Y) Z \\
& -\left(\frac{r}{3}+3 f^{2}+2 f^{\prime}\right)(\eta(X)(\xi \wedge Y) Z+\eta(Y)(X \wedge \xi) Z), \\
S(X, Y) & =\left(\frac{r}{2}+f^{2}+f^{\prime}\right) g(X, Y)-\left(\frac{r}{3}+3 f^{2}+2 f^{\prime}\right) \eta(X) \eta(Y),
\end{aligned}
$$

where $r$ is the scalar curvature and $f^{\prime}=\xi f$.

On a manifold $\widetilde{M}$, for a $(0, k)$-type tensor field $T(k \geq 1)$ and a $(0,2)$-type tensor field $E$, we denote by $Q(E, T)$ a $(0, k+2)$-type tensor field [12] defined as follows

$$
\begin{aligned}
Q(E, T)\left(X_{1}, X_{2}, \ldots, X_{k} ; X, Y\right)= & -T\left(\left(X \wedge_{E} Y\right) X_{1}, X_{2}, \ldots, X_{n}\right) \\
& -T\left(X_{1},\left(X \wedge_{E} Y\right) X_{2}, \ldots, X_{k}\right)-\ldots \\
& -T\left(X_{1}, \ldots,\left(X \wedge_{E} Y\right) X_{k}\right)
\end{aligned}
$$

where $\left(X \wedge_{E} Y\right) Z=E(Y, Z) X-E(X, Z) Y$.

The submanifold $M$ of $\widetilde{M}$ is pseudo parallel ([1], [2], [9]) if

$$
\widetilde{R}(X, Y) \cdot h=\left(\widetilde{\nabla}_{X} \widetilde{\nabla}_{Y}-\widetilde{\nabla}_{Y} \widetilde{\nabla}_{X}-\widetilde{\nabla}_{[X, Y]}\right) h=L_{1} Q(g, h)
$$

for any vector field $X, Y$ tangent to $M$ and $L_{1}$ is a function on the subset $U$ on $M$, where $U=\{x \in M: Q(g, h) \neq 0$ at $x\}$. Again if $L_{1}=0$, then the manifold is said to be semiparallel [15]. The submanifold is Ricci generalized pseudoparallel [19] if its second fundamental form $h$ satisfies

$$
\widetilde{R}(X, Y) \cdot h=L_{2} Q(S, h),
$$

where $L_{2}$ is a function on the subset $V$ of $M$, where $V=\{x \in M: Q(S, h) \neq 0$ at $x\}$. 
3. $f$-Kenmotsu manifolds with respect to quarter symmetric metric connection

Let $\widetilde{\nabla}$ and $\bar{\nabla}$ be the Levi-Civita and quarter symmetric metric connections of an $f$-Kenmotsu manifold $\widetilde{M}$ of dimension $(2 n+1)$. Then we have [11]

$$
\tilde{\nabla}_{X} Y=\widetilde{\nabla}_{X} Y+U(X, Y),
$$

where $U(X, Y)$ is $(1,1)$ tensor field and $X, Y \in \chi(\widetilde{M})$. The tensor $U$ is defined by

$$
U(X, Y)=\frac{1}{2}\left(T(X, Y)+T^{\prime}(X, Y)+T^{\prime}(Y, X)\right),
$$

where

$$
g\left(T^{\prime}(X, Y), Z\right)=g(T(Z, X), Y)
$$

for $X, Y, Z \in \chi(\widetilde{M})$.

Now from (1.2) and (3.3) we infer that

$$
T^{\prime}(X, Y)=g(X, \phi Y) \xi-\eta(X) \phi(Y) .
$$

Using (1.2) and (3.4) in (3.2), we obtain

$$
U(X, Y)=-\eta(X) \phi(Y) .
$$

Therefore, the relation between quarter symmetric metric connection $\bar{\nabla}$ and the Levi-Civita connection $\widetilde{\nabla}$ in an $f$-Kenmotsu manifold is given by

$$
\bar{\nabla}_{X} Y=\widetilde{\nabla}_{X} Y-\eta(X) \phi(Y) \text {. }
$$

Let $\overline{\tilde{R}}$ be the curvature tensor of an $f$-Kenmotsu manifold $\widetilde{M}$ with respect to quarter symmetric metric connection $\bar{\nabla}$. Then $\bar{R}$ is defined by

$$
\overline{\tilde{R}}(X, Y) Z=\overline{\tilde{\nabla}}_{X} \tilde{\tilde{\nabla}}_{Y} Z-\overline{\tilde{\nabla}}_{Y} \overline{\tilde{\nabla}}_{X} Z-\overline{\tilde{\nabla}}_{[X, Y]} Z .
$$

With the help of (2.2), (2.3) and (3.6) we obtain

$$
\begin{aligned}
\tilde{\nabla}_{X} \tilde{\nabla}_{Y} Z & =\widetilde{\nabla}_{X} \widetilde{\nabla}_{Y} Z-\eta(X) \phi\left(\widetilde{\nabla}_{Y} Z\right)-\left(g\left(\widetilde{\nabla}_{X} Y, \xi\right)+f g(X, Y)\right. \\
& -f \eta(X) \eta(Y)) \phi(Z)-\eta(Y)\left(\widetilde{\nabla}_{X} \phi(Z)-\eta(X) Z-\eta(X) \eta(Z) \xi\right)
\end{aligned}
$$

and

$$
\begin{aligned}
\overline{\tilde{\nabla}}_{Y} \overline{\tilde{\nabla}}_{X} Z & =\widetilde{\nabla}_{Y} \widetilde{\nabla}_{X} Z-\eta(Y) \phi\left(\widetilde{\nabla}_{X} Z\right)-\left(g\left(\widetilde{\nabla}_{Y} Z, \xi\right)+f g(X, Y)\right. \\
& -f \eta(X) \eta(Y)) \phi(Z)-\eta(X)\left(\widetilde{\nabla}_{Y} \phi Z-\eta(Y) Z-\eta(Y) \eta(Z) \xi\right)
\end{aligned}
$$

and

$$
\bar{\nabla}_{[X, Y]} Z=\widetilde{\nabla}_{[X, Y]} Z-\eta\left(\widetilde{\nabla}_{X} Y-\widetilde{\nabla}_{Y} X\right) \phi(Z) .
$$


Using these results in (3.7) we have

$$
\begin{aligned}
\overline{\tilde{R}}(X, Y) Z & =\tilde{R}(X, Y) Z+f(\eta(Y) \phi(X)-\eta(X) \phi(Y)) \eta(Z) \\
& +f(\eta(X) g(\phi Y, Z)-\eta(Y) g(\phi X, Z)) \xi
\end{aligned}
$$

where $\tilde{R}$ is curvature tensor with respect to Levi-Civita connection.

Let $\bar{S}$ and $\tilde{S}$ be Ricci curvature tensors of $\widetilde{M}$ with respect to quarter symmetric and Levi-Civita connections. Then $\overline{\tilde{S}}$ is defined by

$$
\overline{\tilde{S}}(X, Y)=\sum_{i=1}^{2 n+1} g\left(\overline{\tilde{R}}\left(e_{i}, X\right) Y, e_{i}\right)
$$

where $\left\{e_{1}, e_{2}, \ldots, e_{2 n+1}\right\}$ is a local orthonormal basis on $\widetilde{M}$. Using the relations in (2.1) and (3.8) we have

$$
\overline{\tilde{S}}(X, Y)=\tilde{S}(X, Y)+f g(\phi X, Y) .
$$

Let $\bar{Q}$ and $\tilde{Q}$ be the Ricci operators on $\widetilde{M}$ with respect to the connections $\bar{\nabla}$ and $\tilde{\nabla}$ respectively. Then using (3.10) we have

$$
\overline{\tilde{Q}} X=\tilde{Q} X+f \phi X .
$$

Let $\overline{\tilde{r}}$ and $\tilde{r}$ be the scalar curvature in $\widetilde{M}$ with respect to the connections $\bar{\nabla}$ and $\tilde{\nabla}$ respectively. Then

$$
\overline{\tilde{r}}=\tilde{r} .
$$

Now for $X, Y \in \chi(\widetilde{M})$ we obtain from the previous results

$$
\begin{gathered}
\overline{\tilde{R}}(X, Y) \xi=\tilde{R}(X, Y) \xi+f(\eta(Y) \phi(X)-\eta(X) \phi(Y)), \\
\overline{\tilde{S}}(X, \xi)=\tilde{S}(X, \xi) \\
\overline{\tilde{Q}} X=\tilde{Q} X .
\end{gathered}
$$

Now we prove the following:

Theorem 3.1. In an $f$-Kenmotsu manifold $\widetilde{M}$ with respect to quarter symmetric metric connection $\bar{\nabla}$ we have

$$
\overline{\tilde{R}}(X, Y) Z+\overline{\tilde{R}}(Y, Z) X+\overline{\tilde{R}}(Z, X) Y=0 .
$$

Proof. Using (3.8) we obtain the theorem. 


\section{Invariant submanifolds of $f$-Kenmotsu manifolds with respect to quarter symmetric metric connection}

Let $M$ be a $(2 m+1)$-dimensional invariant submanifold of a $f$-Kenmotsu manifold $\widetilde{M}$ of dimension $(2 n+1)$ (where $n>m)$. Generally the submanifold $M$ is said to be invariant submanifold of $\widetilde{M}$ if $\phi(T M) \subset T M$. Let $\widetilde{\nabla}$ and $\overline{\tilde{\nabla}}$ be the Levi-Civita and quarter symmetric metric connections of $\widetilde{M}$. Let $\nabla$ and $\bar{\nabla}$ be the induced connections on $M$ form the connections $\widetilde{\nabla}$ and $\bar{\nabla}$.

Let $h$ and $\bar{h}$ be the second fundamental forms of the submanifold with respect to Levi-Civita connections and quarter symmetric metric connections respectively.

Then we have

$$
\overline{\tilde{\nabla}}_{X} Y=\bar{\nabla}_{X} Y+\bar{h}(X, Y) .
$$

Using the equation (3.6) in (4.1) we have

$$
\bar{\nabla}_{X} Y+\bar{h}(X, Y)=\nabla_{X} Y+h(X, Y)-\eta(X) \phi(Y)
$$

Since the submanifold is invariant, therefore comparing tangential and normal components, we have

$$
\begin{gathered}
\bar{\nabla}_{X} Y=\nabla_{X} Y-\eta(X) \phi(Y), \\
\bar{h}(X, Y)=h(X, Y) .
\end{gathered}
$$

Thus the second fundamental forms $\bar{h}$ and $h$ of the submanifold with respect to the quarter symmetric metric connection and the Levi-Civita connection are the same. From (3.6) and (4.3) we can say that an invariant submanifold admits quarter symmetric connections. Hence we have the following:

Lemma 4.1. Let $M$ be an invariant submanifold of a $f$-Kenmotsu manifold $\widetilde{M}$, and $\widetilde{\nabla}$ and $\overline{\tilde{\nabla}}$ are the Levi-Civita and quarter symmetric metric connections of $\widetilde{M}$. If $\nabla$ and $\bar{\nabla}$ are the induced connections on $M$ form the connections $\widetilde{\nabla}$ and $\overline{\tilde{\nabla}}$ of $\widetilde{M}$ respectively, then $M$ admits a quarter symmetric metric connection and the second fundamental forms with respect to $\widetilde{\nabla}$ and $\overline{\tilde{\nabla}}$ are same.

Theorem 4.1. Any invariant submanifold of an $f$-Kenmotsu manifold is totally geodesic with respect to the Levi-Civita connections if and only if it is so with respect to quarter symmetric metric connections.

Proof. The above theorem follows from the Lemma 4.1.

Using (2.8) and (4.4), we can say that the mean curvature vector with respect to the Levi-Civita connection and quarter symmetric metric connection are same. Thus we have the following:

Theorem 4.2. Let $M$ be an invariant submanifold of an $f$-Kenmotsu manifold $\widetilde{M}$. Then the mean curvature vector with respect to the Levi-Civita connection and quarter symmetric metric connection are same.

We may state the following:

Corollary 4.1. An invariant submanifold of a $f$-Kenmotsu manifold is totally umbilical with respect to the Levi-Civita connection if and only if it is totally umbilical 
with respect to the quarter symmetric metric connection.

Corollary 4.2. An invariant submanifold of a $f$-Kenmotsu manifold is minimal with respect to the Levi-Civita connection if and only if it is minimal with respect to the quarter symmetric metric connection.

Example 4.1. We consider a five-dimensional manifold $\widetilde{M}=\left\{\left(x_{1}, x_{2}, x_{3}, x_{4}, t\right) \in\right.$ $\left.\mathbb{R}^{5}: t \neq 0\right\}$, where $\left(x_{1}, x_{2}, x_{3}, x_{4}, t\right)$ are the standard coordinates in $\mathbb{R}^{5}$. Let us choose the vector fields

$$
e_{1}=t^{2} \frac{\partial}{\partial x_{1}}, \quad e_{2}=t^{2} \frac{\partial}{\partial x_{2}}, \quad e_{3}=t^{2} \frac{\partial}{\partial x_{3}}, \quad e_{4}=t^{2} \frac{\partial}{\partial x_{4}}, \quad e_{5}=\frac{\partial}{\partial t},
$$

which are linearly independent at each point of $\widetilde{M}$. We define the metric $g$ such that $\left\{e_{1}, e_{2}, e_{3}, e_{4}, e_{5}\right\}$ is an orthonormal basis of $\widetilde{M}$ i.e.,

$$
\begin{aligned}
g\left(e_{i}, e_{j}\right) & =1 \quad \text { if } i=j \\
& =0 \quad \text { if } i \neq j, \text { where } \quad 1 \leqslant i, j \leqslant 5 .
\end{aligned}
$$

We consider a 1-form $\eta$ defined by

$$
\eta(X)=g\left(X, e_{5}\right), \quad X \in \chi(\widetilde{M}) .
$$

That is, we choose $e_{5}=\xi$. We define the tensor field $\phi$ by

$$
\phi\left(e_{1}\right)=-e_{2}, \quad \phi\left(e_{2}\right)=e_{1}, \quad \phi\left(e_{3}\right)=-e_{4}, \quad \phi\left(e_{4}\right)=e_{3}, \quad \phi\left(e_{5}\right)=0 .
$$

The linearity property of $g$ and $\phi$ shows that

$$
\begin{gathered}
\eta\left(e_{5}\right)=1, \quad \phi^{2}(X)=-X+\eta(X) e_{5}, \\
g(\phi X, \phi Y)=g(X, Y)-\eta(X) \eta(Y),
\end{gathered}
$$

for any vector fields $X, Y$ on $\widetilde{M}$. Then $\widetilde{M}(\phi, \xi, \eta, g)$ forms an almost contact manifold with $e_{5}=\xi$. Let $\tilde{\nabla}$ be the Levi-Civita connection with respect to the metric $g$. Then we have

$$
\left[e_{5}, e_{i}\right]=\frac{2}{t} e_{i}, \quad i=1,2,3,4, \quad \text { and } \quad\left[e_{i}, e_{j}\right]=0, \quad \text { otherwise. }
$$

Now by Koszul's formula, we can obtain the following

$$
\begin{aligned}
& \tilde{\nabla}_{e_{1}} e_{1}=\frac{2}{t} e_{5}, \quad \tilde{\nabla}_{e_{1}} e_{5}=-\frac{2}{t} e_{1}, \quad \tilde{\nabla}_{e_{2}} e_{5}=-\frac{2}{t} e_{2}, \quad \tilde{\nabla}_{e_{2}} e_{2}=\frac{2}{t} e_{5}, \\
& \tilde{\nabla}_{e_{3}} e_{3}=\frac{2}{t} e_{5}, \quad \tilde{\nabla}_{e_{3}} e_{5}=-\frac{2}{t} e_{3}, \quad \tilde{\nabla}_{e_{4}} e_{4}=\frac{2}{t} e_{5}, \quad \tilde{\nabla}_{e_{4}} e_{5}=-\frac{2}{t} e_{4}, \\
& \tilde{\nabla}_{e_{i}} e_{j}=0 \text {, otherwise. }
\end{aligned}
$$

The above relations imply that the manifold satisfies

$$
\tilde{\nabla}_{X} \xi=f\{X-\eta(X) \xi\}
$$


for $\xi=e_{5}$, and $f=-\frac{2}{t}$. Hence we can say that $\widetilde{M}$ is an $f$-Kenmotsu manifold. Again since $f^{2}+f^{\prime} \neq 0$, so the manifold is regular $f$-Kenmotsu manifold.

Let $M$ be a subset of $\widetilde{M}$ and consider the immersion $h: M \rightarrow \widetilde{M}$ defined by

$$
h\left(x_{1}, x_{2}, t\right)=\left(x_{1}, x_{2}, 0,0, t\right) .
$$

It is easy to prove that $M=\left\{\left(x_{1}, x_{2}, t\right) \in \mathbb{R}^{3}: t \neq 0\right\}$ is a submanifold of $\widetilde{M}$, where $\left(x_{1}, x_{2}, t\right)$ are the standard coordinates of $\mathbb{R}^{3}$. We choose the vector fields

$$
e_{1}=t^{2} \frac{\partial}{\partial x_{1}}, \quad e_{2}=t^{2} \frac{\partial}{\partial x_{2}}, \quad e_{5}=\frac{\partial}{\partial t} .
$$

We define $g_{1}$ such that $\left\{e_{1}, e_{2}, e_{5}\right\}$ is an orthonormal basis of $M$. That is,

$$
\begin{aligned}
& g_{1}\left(e_{i}, e_{j}\right)=1 \quad \text { if } i=j \\
& =0 \quad \text { if } i \neq j \text {, where } i, j=1,2,5 \text {. }
\end{aligned}
$$

We define a 1 -form $\eta_{1}$ and a $(1,1)$ tensor $\phi_{1}$ respectively by

$$
\eta_{1}=g_{1}\left(X, e_{5}\right), \quad \text { and } \quad \phi_{1}\left(e_{1}\right)=-e_{2}, \quad \phi_{1}\left(e_{2}\right)=e_{1}, \quad \phi_{1}\left(e_{5}\right)=0 .
$$

The linearity property of $g_{1}$ and $\phi_{1}$ shows that

$$
\begin{gathered}
\eta_{1}\left(e_{5}\right)=1, \quad \phi_{1}^{2}(X)=-X+\eta_{1}(X) e_{5}, \\
g_{1}\left(\phi_{1} X, \phi_{1} Y\right)=g_{1}(X, Y)-\eta_{1}(X) \eta_{1}(Y)
\end{gathered}
$$

for any vector fields $X, Y$ on $M\left(\phi_{1}, \xi, \eta_{1}, g_{1}\right)$. It is seen that $M$ is an invariant submanifold of $\widetilde{M}$ with $e_{5}=\xi$. Moreover, let $\nabla$ be the Levi-Civita connection with respect to the metric $g_{1}$. Then we have

$$
\left[e_{5}, e_{i}\right]=\frac{2}{t} e_{i}, \quad i=1,2, \quad \text { and } \quad\left[e_{i}, e_{j}\right]=0, \quad \text { otherwise. }
$$

Now by Koszul's formula, we can obtain the following

$$
\begin{gathered}
\nabla_{e_{1}} e_{1}=\frac{2}{t} e_{5}, \quad \nabla_{e_{1}} e_{5}=-\frac{2}{t} e_{1}, \quad \nabla_{e_{2}} e_{5}=-\frac{2}{t} e_{2}, \quad \nabla_{e_{2}} e_{2}=\frac{2}{t} e_{5}, \\
\nabla_{e_{i}} e_{j}=0, \quad \text { otherwise. }
\end{gathered}
$$

Let us consider $\overline{\tilde{\nabla}}$ and $\bar{\nabla}$ be the quarter symmetric metric connections on $\widetilde{M}$ and $M$ respectively. Using (3.6) we can find $\overline{\tilde{\nabla}}_{e_{i}} e_{j}$ and $\bar{\nabla}_{e_{i}} e_{j}$.

Let $h$ and $\bar{h}$ be the second fundamental forms with respect to Levi-Civita connection and quarter symmetric metric connections. By using (2.4) we have

$$
h(X, Y)=0, \quad \text { and } \quad \bar{h}(X, Y)=0
$$

for any vector field on the manifold. Thus the submanifold is totally geodesic with respect to Levi-Civita connection and quarter symmetric metric connection. Hence the Theorem 4.1 is verified. 


\section{Invariant submanifolds of $f$-Kenmotsu manifolds with certain curvature conditions on the second fundamental form}

Now from (2.3) and (2.4) we have

$$
\nabla_{X} \xi+h(X, \xi)=f(X-\eta(X) \xi) .
$$

Comparing normal and tangential components, we have

$$
\begin{gathered}
h(X, \xi)=0, \\
\nabla_{X} \xi=f(X-\eta(X) \xi) .
\end{gathered}
$$

Using (4.4) and (5.2) we can say that

$$
\bar{h}(X, \xi)=0 .
$$

From (2.2) and (2.4), we obtain

$$
\left(\nabla_{X} \phi\right) Y-h(X, \phi Y)+\phi(h(X, Y))=f(g(\phi X, Y) \xi-\eta(Y) \phi X) .
$$

Comparing tangential components, we get

$$
h(X, \phi Y)=\phi(h(X, Y)) .
$$

Theorem 5.1. Let $M$ be an invariant submanifold of an $f$-Kenmotsu manifold $\widetilde{M}$. Then $h$ is recurrent with respect to the quarter symmetric metric connection if and only if it is totally geodesic. Proof. If $h$ is recurrent with respect to quarter symmetric metric connection, then from (2.11) we have

$$
\left(\tilde{\nabla}_{X} h\right)(Y, Z)=\pi(X) h(Y, Z) .
$$

Putting $Z=\xi$ and using (5.2) we have

$$
h\left(Y, \bar{\nabla}_{X} \xi\right)=0 .
$$

From (2.3), (5.2) and the above equation we obtain $f h(X, Y)=0$. Consequently $h(X, Y)=0$, for any $X, Y \in \chi(M)$. The converse is trivial.

This proves the theorem.

Theorem 5.2. Let $M$ be an invariant submanifold of a f-Kenmotsu manifold $\widetilde{M}$. Then $M$ has parallel third fundamental form with respect to the quarter symmetric metric connection if and only if it is totally geodesic.

Proof. Let $M$ has parallel third fundamental form with respect to quarter symmetric metric connection. Then we have

$$
\left(\tilde{\nabla}_{X} \bar{\nabla}_{Y} h\right)(Z, W)=0 .
$$


Substituting $W=Z=\xi$ and using the equations (2.1), (5.2) we have from above

$$
2 h\left(\bar{\nabla}_{X} \xi, \bar{\nabla}_{Y} \xi\right)=0 .
$$

Now we use the result in (2.3) and we get $f^{2} h(X, Y)=0$, thus we have $h(X, Y)=0$, for any $X, Y \in \chi(M)$. Therefore, $M$ is totally geodesic. The converse statement is trivially true.

This completes the proof.

Theorem 5.3. Let $M$ be an invariant submanifold of an $f$-Kenmotsu manifold $\widetilde{M}$. Then $h$ is 2-recurrent with respect to the quarter symmetric metric connection if and only if it is totally geodesic.

Proof. Let $h$ be 2-recurrent with respect to quarter symmetric metric connection. Then from (2.12) we have

$$
\left(\tilde{\nabla}_{X} \bar{\nabla}_{Y} h\right)(Z, W)=\psi(X, Y) h(Z, W) .
$$

Putting $Z=\xi$ and using the equation (5.2) we have

$$
\left(\bar{\nabla}_{X} \bar{\nabla}_{Y} h\right)(\xi, W)=0 .
$$

Then by previous theorem we can say $M$ is totally geodesic. The converse is trivially true.

This finishes the proof.

Theorem 5.4. An invariant submanifold of an $f$-Kenmotsu manifold is totally geodesic if and only if $Q\left(S, \bar{\nabla}_{X} h\right)=0$, provided $f^{2} \neq \xi f$.

Proof. Let $M$ be an invariant submanifold of an $f$-Kenmotsu manifold $\widetilde{M}$ satisfying $Q\left(S, \overline{\tilde{\nabla}}_{X} h\right)=0$. Then

$$
Q\left(S, \overline{\tilde{\nabla}}_{X} h\right)(W, K ; U, V)=0
$$

for the vector fields $X, W, K, U, V \in \chi(M)$. By the above equation and (2.20), we have

$$
\begin{aligned}
0= & -\left(\overline{\tilde{\nabla}}_{X} h\right)(S(V, W) U, K)+\left(\overline{\tilde{\nabla}}_{X} h\right)(S(U, W) V, K) \\
& -\left(\tilde{\nabla}_{X} h\right)(W, S(V, K) U)+\left(\tilde{\nabla}_{X} h\right)(W, S(U, K) V) .
\end{aligned}
$$

Hence,

$$
\begin{aligned}
0= & -\bar{\nabla}_{X}^{\perp} h(S(V, W) U, K)+h\left(\bar{\nabla}_{X} S(V, W) U, K\right)+h\left(S(V, W) U, \nabla_{X} K\right) \\
& +\bar{\nabla}_{X}^{\perp} h(S(U, W) V, K)-h\left(\bar{\nabla}_{X} S(U, W) V, K\right)-h\left(S(U, W) V, \bar{\nabla}_{X} K\right) \\
& -\bar{\nabla}_{X}^{\perp} h(W, S(V, K) U)+h\left(\bar{\nabla}_{X} W, S(V, K) U\right)+h\left(W, \bar{\nabla}_{X} S(V, K) U\right) \\
& +\bar{\nabla}_{X}^{\perp} h(W, S(U, K) V)-h\left(\bar{\nabla}_{X} W, S(U, K) V\right)-h\left(W, \bar{\nabla}_{X} S(U, K) V\right) .
\end{aligned}
$$

Substituting $K=V=W=\xi$ in the above equation and using equation (5.2) we can obtain

$$
S(\xi, \xi) h\left(U, \bar{\nabla}_{X} \xi\right)=0 .
$$


Using the equations (2.3), (2.15) in the above equation, we have

$$
(2 n)\left(f^{2}-\xi f\right) f h(U, \phi X)=0 .
$$

With the help of (5.6) we obtain $h(U, X)=0$, provided $f^{2} \neq \xi f$, for any $U, X \in$ $\chi(M)$. Hence the submanifold is totally geodesic. Converse is trivially true.

This proves the theorem.

Theorem 5.5. Let $M$ an invariant submanifold of an $f$-Kenmotsu manifold $\widetilde{M}$. Then $M$ is totally geodesic if and only if the submanifold is semiparallel with respect to quarter symmetric connection, provided $f^{2} \neq \xi f$.

Proof. If the submanifold $M$ is semiparallel then

$$
\overline{\tilde{R}}(X, Y) h(U, V)=0 .
$$

The above equation gives

$$
R^{N}(X, Y) h(U, V)-h(\bar{R}(X, Y) U, V)-h(U, \bar{R}(X, Y) V)=0 .
$$

Putting $U=X=\xi$ in the forgoing equation and using (5.2) we have

$$
h(\bar{R}(\xi, Y) \xi, V)=0 .
$$

Then using (3.13) we get

$$
\left\{f^{2}-\xi f\right\} h(Y, V)=0 .
$$

With the help of (5.6) we obtain $h(Y, V)=0$, provided $f^{2} \neq \xi f$, for any $Y, V \in$ $\chi(M)$. Hence the submanifold is totally geodesic. Converse is trivially true.

This completes the proof.

\section{REF E R E N C E S}

1. A. C. Asperti, G. A. Lobos and F. Mercuri, Pseudo-parallel immersions in space forms, Mat. Contemp., 17(1999), 53-70.

2. A. C. Asperti, G. A. Lobos and F. Mercuri, Pseudo-parallel submanifolds of a space forms, Adv. Geom. 2(2002), 57-71.

3. S. C. Biswas and U. C. De Quarter-symmetric metric connection in an SP-Sasakian manifold, Commun. Fac. Sci. Univ. Ank. Series, 46(1997), 49-56.

4. C. Calin and M. Crasmareanu, From the Eisenhart problem to Ricci solitons in $f$-Kenmotsu manifolds, Bulletin of the Malaysian Mathematical Sciences Society, 33(2010), 361-368.

5. B. Y. Chen, Geometry of submanifolds, Maecel Dekker Inc., New York (1973).

6. T. Demirli, C. Ekici and A. Gorgulu, Ricci solitons in f-Kenmotsu manifolds with the semi-symmetric non-metric connection, New Trends in Mathematical Sci., 4(2016), 276-284. 
7. U. C. De, P. Zhao, K. Mandal and Y. Han, Certain conditions on P-Sasakian manifolds admitting a quarter-symmetric metric connection, Chin. Ann. Math. Ser. B, 41(2020), 133-146.

8. U. C. De and P. Majhi, On invariant submanifolds of Kenmotsu manifolds, J. Geom., 106(2015), 109-122.

9. R. Deszcz, On pesudosymmetric spaces, Bull. Soc. Belg. Math. Ser A, 44(1992), 1-34.

10. A. Friedman and J. A. Schouten, Uber die geometric derhalbsymmetrischen Ubertragung, Math. Zeitscr., 21(1924), 211-223.

11. S. Golab, On a semi-symmetric and quarter symmetric linear connections, Tensor, N. S., 29(1975), 249-254.

12. C. Hu and Y. Wang, A note on invariant Submanifolds of trans-Sasakian manifolds, Int. Ele. J. of Geom., 9(2016), 27-35.

13. Janssens, D. and Vanhecke, L., Almost contact structure and curvature tensor, Kodai Math. J. 4(1981), 1-27.

14. M. Kon, Invariant submanifolds of normal contact metric manifolds, Kodai Math. Sem. Repors, 25(1973), 330-336.

15. U. Lumiste, Semiparallel submanifolds in space forms, Springer Science + Business Media, LLC, 2009, DOI: 10.1007/978-0-387-49913-0.

16. R. S. Mishra and S. N. Pandey, On quarter-symmetric metric F-connections, Tensor, N.S., 34(1980), 1-7.

17. A. K. Mondal and U. C. De, Some properties of a quarter-symmetric connection on a Sasakian manifold, Bull of Math. Anal. and Appl., 3(2009), 99-108.

18. S. Mukhopadhyay, A. K. Roy, and B. Barua, Some properties of a quarter-symmetric metric connection on a Riemannian manifold, Soochow J. of Math., 17(1991), 205-211.

19. C. Murathan, K. Arslan and E. Ezentas, Ricci generalizespseudo-symmetric immersions, Differential geometry and its applications, Matfyzpress, Prague, 99-108(2005).

20. C. Ozgur and C. Murathan, On invariant submanifolds of Lorentzian Para-Sasakian manifolds, Arab. J. Sci. Eng., 34(2008), 177-185.

21. C. Ozgur, S. Sular and C. Murathan, On pseudoparallel invariant submanifolds of contact metric manifolds, Bull. Transilv Univ. Brasov Ser. B(N.S), 14(2007), 227-234.

22. Z. Olszak and R. Rosca, Normal locally conformal almost cosymplectic manifolds, Publicationes Mathematicae Debrecen, 39(1991), 315-323.

23. W. Roter, On conformally recurrent Ricci-recurrent manifolds, Colloq Math., 46(1982), 45-57.

24. A. Sarkar, N. Biswas and M. Sen, On some submanifolds of ( $\epsilon$ )-LP-Sasakian manifolds, Acta Univ. Apu., 61(2020), 65-80.

25. A. Sarkar and M. Sen, On invariant submanifolds of trans-Sasakian manifolds, Proceedings Estonian Academy of Sciences, 61(2012), 29-37.

26. S. Sular, C. Ozgur, and U. C. De, Quarter-symmetric metric connection in a Kenmotsu manifold, SUT J. of Math., 44(2008), 297-306.

27. K. Yano, and M. Kon, Structures on manifolds, World Scientific pub., 1984.

28. K. Yano,and T. Imai, Quarter-symmetric metric connections and their curvature tensors, Tensor, N.S. 38(1982), 13-18. 
29. A. Yildiz, U. C. De and M. Turan, On 3-dimensional $f$-Kenmotsu manifolds and Ricci solitons, Ukrainian Mathematical J., 65(2013), 620-628.

30. A. Yildiz and C. Murathan, Invariant submanifold of Sasakian space forms, J. Geom. 95(2009), 135-150.

\author{
Avijit Sarkar \\ Department of Mathematics \\ University of Kalyani \\ Kalyani 741235 \\ West Bengal \\ India \\ avjaj@yahoo.co.in \\ Nirmal Biswas \\ Department of Mathematics \\ University of Kalyani \\ Kalyani 741235 \\ West Bengal \\ India \\ nirmalbiswas.maths@gmail.com
}

